\title{
ON ERGODIC PROBLEM FOR HAMILTON-JACOBI-ISAACS EQUATIONS
}

\author{
Piernicola Bettiol ${ }^{1}$
}

\begin{abstract}
We study the asymptotic behavior of $\lambda v_{\lambda}$ as $\lambda \rightarrow 0^{+}$, where $v_{\lambda}$ is the viscosity solution of the following Hamilton-Jacobi-Isaacs equation (infinite horizon case)

$$
\lambda v_{\lambda}+H\left(x, D v_{\lambda}\right)=0,
$$

with

$$
H(x, p):=\min _{b \in B} \max _{a \in A}\{-f(x, a, b) \cdot p-l(x, a, b)\} .
$$

We discuss the cases in which the state of the system is required to stay in an $n$-dimensional torus, called periodic boundary conditions, or in the closure of a bounded connected domain $\Omega \subset \mathbb{R}^{n}$ with sufficiently smooth boundary. As far as the latter is concerned, we treat both the case of the Neumann boundary conditions (reflection on the boundary) and the case of state constraints boundary conditions. Under the uniform approximate controllability assumption of one player, we extend the uniform convergence result of the value function to a constant as $\lambda \rightarrow 0^{+}$to differential games. As far as state constraints boundary conditions are concerned, we give an example where the value function is Hölder continuous.
\end{abstract}

Mathematics Subject Classification. 35B40, 49L25, 49N70.

Received January 20, 2004. Revised November 3, 2004.

\section{INTRODUCTION}

We investigate the limiting behavior of the viscosity solution $v_{\lambda}$ of the following stationary Hamilton-JacobiIsaacs (HJI for short) equation (infinite horizon case)

where

$$
\lambda v_{\lambda}+H\left(x, D v_{\lambda}\right)=0,
$$

$$
H(x, p):=\min _{b \in B} \max _{a \in A}\{-f(x, a, b) \cdot p-l(x, a, b)\}
$$

is called Hamiltonian function and the constant $\lambda>0$ is called discount factor. Here, $A$ and $B$ are compact metric spaces and $x$ belongs to a closed set $\bar{\Omega} \subset \mathbb{R}^{n}$, that we will specify later. The function $f$ takes values in $\mathbb{R}^{n}$, it is bounded and Lipschitz continuous in all variables, while $l$ is a bounded real-valued Lipschitz continuous function. We discuss the cases in which the state of the system is required to stay in an $n$-dimensional torus, $\bar{\Omega}=\mathbb{T}^{n}$, called periodic boundary conditions (B.C.), or in the closure of a bounded connected domain $\Omega \subset \mathbb{R}^{n}$

Keywords and phrases. Hamilton-Jacobi-Isaacs equations, viscosity solutions, asymptotic behavior, differential games, boundary conditions, ergodicity.

1 SISSA/ISAS via Beirut, 2-4 - 34013 Trieste, Italy; bettiol@ma.sissa.it 
with a sufficiently smooth boundary $\partial \Omega$. As far as the latter is concerned, we treat the case of the Neumann B.C. (case of reflection on the boundary) and an example of state constraints B.C. The existence and the uniqueness results for the HJI equations with the above conditions have been obtained in the Viscosity Solutions framework: see the textbooks of Bardi and Capuzzo-Dolcetta [8] and Barles [10] for general results (cf. also [17-20]; while for the Neumann B.C. and for the state constraints B.C. we refer to the articles of Lions [30] and Koike [27], respectively.

The state constraints problem was first treated via viscosity solutions approach by Soner in [34] for deterministic optimal control problems, where the associated value function satisfies first order Hamilton-Jacobi-Bellman PDE's. In this paper, he proposed an appropriate boundary condition for the PDE's under which the value function is the unique continuous viscosity solution. For further development see [5, 13, 32] for single player problems and [24] for the general setting of differential inclusions. More recently, in [27] Koike gives representation formulas for the viscosity solution of the Hamilton-Jacobi-Isaacs equation with infinite horizon and he proves the uniqueness of the solutions under natural boundary conditions. For pursuit-evasion differential games with state constraints, we refer to the articles $[9,14]$.

Here, we use an approach developed in the author's joint work with Cardaliaguet and Quincampoix [12], where a Bolza problem for state constrained differential games is treated. By suitably applying some adapted results, we are able to obtain the Hölder regularity of the value function in our state-constrained example: this is crucial for our proof of the ergodic property. The main idea is to apply a selection argument for strategies combined with a $L^{1}$-estimate on these strategies.

By $v(x, t)$ we denote the solution of the time dependent HJI equation (finite horizon case)

$$
\partial_{t} v(x, t)+H\left(x, D_{x} v(x, t)\right)=0
$$

with the boundary condition $v(x, 0)=0$.

We remind the reader that finding the solutions of the above HJI equations (1.1) and (1.2) corresponds to studying the differential game problems that follow. Let us denote the set of the controls of the first player and of the second player by $\mathcal{A}:=\{a:[0,+\infty[\longrightarrow A$ measurable $\}$ and $\mathcal{B}:=\{b:[0,+\infty[\longrightarrow B$ measurable $\}$, respectively. Once the controls $a(\cdot) \in \mathcal{A}$ and $b(\cdot) \in \mathcal{B}$ are fixed, for the periodic B.C. the ODE is

$$
\left\{\begin{array}{l}
\dot{y}(t)=f(y(t), a(t), b(t)) \\
y(0)=x \quad x \in \mathbb{T}^{n}
\end{array}\right.
$$

where $\mathbb{T}^{n}=\mathbb{R}^{n} / \mathbb{Z}^{n} ;$ while in the Neumann type B.C. the ODE is

$$
\left\{\begin{array}{l}
y(t)=x+\int_{0}^{t} f(y(s), a(s), b(s)) \mathrm{d} s-\int_{0}^{t} \gamma(y(s)) \mathrm{d} L_{s} \\
y(t) \in \bar{\Omega}, \forall t \geq 0 ; L_{t} \text { is continuous, nondecreasing on }[0, \infty[ \\
L_{t}=\int_{0}^{t} 1_{\partial \Omega}(y(s)) \mathrm{d} L_{s}, \text { for } t \geq 0 .
\end{array}\right.
$$

Finally, in state constraints B.C. we have the ODE

$$
\begin{cases}\dot{y}(t)=f(y(t), a(t), b(t)) \\ y(0)=x & x \in \bar{\Omega} \\ y(t) \in \bar{\Omega} & \forall t \geq 0\end{cases}
$$

The existence and uniqueness of the solution of the ODE (1.4), called the solution of the Skorokhod problem, can be found in [31] in the more general context of SDE's. For any starting point $x$ we denote by $y_{x}(\cdot)=$ $y_{x}(\cdot, a(\cdot), b(\cdot))$ the solution of the ODE $(1.3),(1.4)$ or $(1.5)$ controlled by $a(\cdot)$ and $b(\cdot)$. 
The value functions $v_{\lambda}(x)$ and $v(x, t)$ are given by

$$
\begin{gathered}
v_{\lambda}(x):=\inf _{\alpha \in \Delta} \sup _{b \in \mathcal{B}} \int_{0}^{\infty} l\left(y_{x}(s), \alpha[b](s), b(s)\right) \mathrm{e}^{-\lambda s} \mathrm{~d} s \quad \lambda>0, x \in \bar{\Omega}, \\
v(x, t):=\inf _{\alpha \in \Delta} \sup _{b \in \mathcal{B}} \int_{0}^{t} l\left(y_{x}(s), \alpha[b](s), b(s)\right) \mathrm{d} s \quad t \geq 0 x \in \bar{\Omega},
\end{gathered}
$$

where $\Delta$ denotes the nonanticipating strategies set of the first player, i.e. the set of the maps $\alpha: \mathcal{B} \longrightarrow \mathcal{A}$ such that if, for any $t>0$ and $b(\cdot), b^{\prime}(\cdot) \in \mathcal{B}, b(s)=b^{\prime}(s) \forall s \leq t$ implies $\alpha[b](s)=\alpha\left[b^{\prime}\right](s) \forall s \leq t$.

Here, we want to study the so-called ergodic problem, namely the convergence (to a constant) of $\lambda v_{\lambda}(x)$ as $\lambda \longrightarrow 0^{+}$and the convergence of the term $\frac{1}{T} v(x, T)$ as $T \longrightarrow \infty$ (cf. Chap. 7 of [8] for an introduction on Hamilton-Jacobi-Bellman equations). There is a relationship between the ergodic problem and the notion of ergodicity in the Dynamical Systems Theory (see also [1-3]). Indeed, let us consider, for a moment, the ordinary differential equations obtained by the controlled systems (1.3), (1.4) or (1.5), where we fix the controls $a(\cdot) \in \mathcal{A}$ and $b(\cdot) \in \mathcal{B}$. In this case the ergodicity is traditionally formulated in terms of measure theory: denoting the evolution of the system as $\phi_{t}(x): x \mapsto y_{x}(t)$ with $t \in \mathbb{R}_{\geq 0}$ from $\bar{\Omega}$ into $\bar{\Omega}$ (where $y_{x}(\cdot)=y_{x}(\cdot, a(\cdot), b(\cdot)$ ) is the solution of (1.3), (1.4) or (1.5)), taking a measure $\mu$ which is invariant under the flow $\phi_{t} \forall t \geq 0$, the system is ergodic with respect to the measure $\mu$ when

$$
\lim _{T \rightarrow \infty} \frac{1}{T} \int_{0}^{T} l\left(y_{x}(t)\right) \mathrm{d} t=\int_{\bar{\Omega}} l(z) \mathrm{d} \mu(z) \quad \text { for } \mu-\text { a.e. } x \in \bar{\Omega}
$$

holds for any function $l \in L^{1}(\bar{\Omega}, \mu)$ (see [16]). By recalling the following known relationship

$$
\lim _{\lambda \rightarrow 0^{+}} \lambda \int_{0}^{\infty} l\left(y_{x}(t)\right) \mathrm{e}^{-\lambda t} \mathrm{~d} t=\lim _{T \rightarrow \infty} \frac{1}{T} \int_{0}^{T} l\left(y_{x}(t)\right) \mathrm{d} t
$$

provided that at least one side is meaningful (Abelian-Tauberian Theorem in [33]), we see that the convergence properties $\lim _{\lambda \rightarrow 0^{+}} \lambda v_{\lambda}(x)$ and $\lim _{T \rightarrow \infty} \frac{1}{T} v(x, T)$ to a constant are related to the ergodic theory of dynamical systems. We study the stronger property of uniform convergence in $\bar{\Omega}$ to a constant, and this is equivalent to the ergodicity property coupled with the uniqueness of the invariant measure (see $[1,2,16]$ ). Such a dynamical system is called uniquely ergodic.

As far as HJI equations are concerned, for Neumann B.C. case, Lions in [30] proves an ergodic theorem under the following assumptions on the Hamiltonian $H(x, p)$ :

$$
H(x, p) \rightarrow \infty \quad \text { as }|p| \rightarrow+\infty, \quad \text { uniformly in } x \in \bar{\Omega}
$$

and $H \in \mathcal{C}\left(\bar{\Omega} \times \mathbb{R}^{n}\right)$. In a different framework (see [1]), Alvarez and Bardi treat the periodic B.C. case using the condition of exact controllability instead of (1.6). Here, we extend the convergence result of the value function $\lambda v_{\lambda}(x)$ for periodic B.C., for Neumann B.C. and in an example for state constraints B.C., under a uniform approximate controllability assumption of the first player. This notion was first introduced by Arisawa in [4] for optimal control problems with a single player. Ergodic optimal stochastic control problems are studied in the article [6].

The paper is organized as follows. Section 2 is devoted to some basic definitions and preliminary assumptions. In Section 3, we treat the periodic and the Neumann B.C. cases; while in Section 4 we deal with the state constraints boundary conditions. In Section 5, we give an Abelian-Tauberian Theorem, that provides equivalent characterizations of the notion of ergodicity. Finally, in the appendix, we treat some estimates concerning the solutions to the Skorokhod problem. 


\section{BASIC DEFINITIONS AND ASSUMPTIONS}

We deal with controlled systems given by (1.3), (1.4) or (1.5) where

$$
\begin{aligned}
f: \mathbb{R}^{n} \times A \times B & \longrightarrow \mathbb{R}^{n} \\
(x, a, b) & \longmapsto f(x, a, b),
\end{aligned}
$$

$A$ and $B$ are compact metric spaces, $\mathcal{A}:=\{a(\cdot):[0,+\infty[\longrightarrow A$ measurable $\}$ and $\mathcal{B}:=\{b(\cdot):[0,+\infty[\longrightarrow$ $B$ measurable $\}$ are the sets of the controls of the first player and of the second player, respectively. As usual, we denote an element of $\mathcal{A}(\operatorname{resp} . \mathcal{B})$ by $a(\cdot)(\operatorname{resp} . b(\cdot))$ or simply by $a($ resp. $b)$ when it is clear we are using measurable functions. As far as the function $f$ is concerned, we assume the existence of a positive constant $M$ such that for any $x, x^{\prime} \in \mathbb{R}^{n}, a, a^{\prime} \in A, b, b^{\prime} \in B$

$$
\left\{\begin{array}{l}
\|f(x, a, b)\|_{\infty} \leq M \\
\left|f(x, a, b)-f\left(x^{\prime}, a^{\prime}, b^{\prime}\right)\right| \leq M\left(\left|x-x^{\prime}\right|+\left|a-a^{\prime}\right|+\left|b-b^{\prime}\right|\right) .
\end{array}\right.
$$

We recall that by $t \mapsto y_{x}(t ; a, b)=y_{x}(t ; a(t), b(t))$ we denote the unique Carathéodory solution of the controlled system. Sometimes, in order to simplify the notations, we simply write $y_{x}(\cdot)$ when the choice of the controls $a(\cdot)$ and $b(\cdot)$ is evident.

We consider games where the first player tries to minimize the cost functional $J_{\lambda}^{\infty}(x, a(\cdot), b(\cdot))$, where for all $T \geq 0$

$$
J_{\lambda}^{T}(x, a(\cdot), b(\cdot)):=\int_{0}^{T} l\left(y_{x}(t), a(t), b(t)\right) \mathrm{e}^{-\lambda t} \mathrm{~d} t,
$$

while the second player tries to maximize $J_{\lambda}^{\infty}$. Here, $\lambda$ is a positive constant and $l: \mathbb{R}^{n} \times A \times B \longrightarrow \mathbb{R}$ is a continuous function satisfying the same regularity conditions of $f$ : $\exists M$ positive constant such that for all $x, x^{\prime} \in \mathbb{R}^{n}, a, a^{\prime} \in A, b, b^{\prime} \in B$

$$
\left\{\begin{array}{l}
|| l(x, a, b) \|_{\infty} \leq M \\
\left|l(x, a, b)-l\left(x^{\prime}, a^{\prime}, b^{\prime}\right)\right| \leq M\left(\left|x-x^{\prime}\right|+\left|a-a^{\prime}\right|+\left|b-b^{\prime}\right|\right) .
\end{array}\right.
$$

We recall the reader that by $\Delta$ we denote the nonanticipating strategies set of the first player: it is the set of the maps $\alpha: \mathcal{B} \longrightarrow \mathcal{A}$ such that if, for any $t>0$ and for any control $b(\cdot)$ and $b^{\prime}(\cdot) \in \mathcal{B}$, which coincides almost everywhere (a.e.) on $[0, t]$, then $\alpha[b](s)=\alpha\left[b^{\prime}\right](s)$ a.e. on $[0, t]$. Analogously we have the nonanticipating strategy set of the second player $\Gamma:=\left\{\beta: \mathcal{A} \longrightarrow \mathcal{B}: a(s)=a^{\prime}(s) \forall s \leq t\right.$ implies $\left.\beta[a](s)=\beta\left[a^{\prime}\right](s) \forall s \leq t\right\}$.

We define the lower and upper values as follows

$$
\begin{aligned}
& v_{\lambda}(x):=\inf _{\alpha \in \Delta} \sup _{b \in \mathcal{B}} \int_{0}^{\infty} l\left(y_{x}(t), \alpha[b](t), b(t)\right) \mathrm{e}^{-\lambda t} \mathrm{~d} t, \\
& u_{\lambda}(x):=\sup _{\beta \in \Gamma} \inf _{a \in \mathcal{A}} \int_{0}^{\infty} l\left(y_{x}(t), a(t), \beta[a](t)\right) \mathrm{e}^{-\lambda t} \mathrm{~d} t .
\end{aligned}
$$

Let us consider the Hamiltonian functions

$$
\begin{aligned}
& H(x, p):=\min _{b \in B} \max _{a \in A}\{-f(x, a, b) \cdot p-l(x, a, b)\} \\
& \tilde{H}(x, p):=\max _{a \in A} \min _{b \in B}\{-f(x, a, b) \cdot p-l(x, a, b)\} .
\end{aligned}
$$


It is well known, through the classical theory, that $v_{\lambda}$ and $u_{\lambda}$ are the viscosity solutions in $\mathbb{R}^{n}$ of the following HJI equations (see $[8,30]$ )

$$
\begin{aligned}
\lambda v_{\lambda}+H\left(x, D v_{\lambda}\right) & =0 \\
\lambda u_{\lambda}+\tilde{H}\left(x, D u_{\lambda}\right) & =0,
\end{aligned}
$$

respectively. Of course, in order to treat the three distinct cases of boundary conditions, we need to add more hypotheses and suitable modifications to the system and to the functions $l$ and $f$. In fact, we will discuss these details below, case by case.

\section{The Periodic and the Neumann type B.C.}

In this section, we prove an ergodic theorem for two classes of spaces:

1) the game state space is an $n$-dimensional torus $\left(\bar{\Omega}=\mathbb{T}^{n}\right)$;

2) the game state space is the closure of a smooth bounded connected domain $\Omega \subset \mathbb{R}^{n}$ and the trajectories of the system are reflected at the boundary $\partial \Omega$.

\subsection{Preliminaries}

The Hamilton-Jacobi-Isaacs equation for the first problem $\left(\bar{\Omega}=\mathbb{T}^{n}\right)$ is coupled with periodic boundary conditions. We suppose that both $f$ and $l$ are periodic in $x$ and the state of the system is defined to be the solution of (1.3).

In the second case, the Hamilton-Jacobi-Isaacs equation is coupled with Neumann boundary conditions:

$$
\frac{D v_{\lambda}}{D \gamma}=0 \quad \text { on } \partial \Omega
$$

where $\gamma=\gamma(x)$ is a smooth vector field on $\partial \Omega$ pointing outwards i.e.

$$
\exists \nu>0 \text { s. t. } \forall x \in \partial \Omega\langle n(x), \gamma(x)\rangle \geq \nu .
$$

In this problem, we suppose that the boundary $\partial \Omega$ is sufficiently smooth such that the distance function from the boundary $\partial \Omega$ itself is at least of class $\mathcal{C}^{1}$ in a neighborhood of $\partial \Omega$; in particular, we suppose that $\partial \Omega \in \mathcal{C}^{1}$ and that $\bar{\Omega}$ satisfies the uniform exterior ball condition, i.e.

$$
\exists r>0 \text { such that } \Omega \cap B(x+r n(x), r)=\emptyset \forall x \in \partial \Omega,
$$

where $n(x)$ denotes the outer unit normal of $\partial \Omega$ at $x$ and $B(z, r)$ is the sphere of $\mathbb{R}^{n}$ with center in $z$ and radius $r$. For conditions on the regularity of the distance function (in a suitable neighborhood of $\bar{\Omega}$ ) we refer the reader, for instance, to the book [25] or to the article [22].

In the second game, once $a(\cdot) \in \mathcal{A}$ and $b(\cdot) \in \mathcal{B}$ are given, the state of the system $y_{x}(t)$ is governed by the following ordinary differential equation with reflection on the boundary

$$
\left\{\begin{array}{l}
y_{x}(t)=x+\int_{0}^{t} f\left(y_{x}(s), a(s), b(s)\right) \mathrm{d} s-\int_{0}^{t} \gamma\left(y_{x}(s)\right) \mathrm{d} L_{s} \\
y_{x}(t) \in \bar{\Omega}, \forall t \geq 0 ; L_{t} \text { is continuous, nondecreasing on }[0, \infty[ \\
L_{t}=\int_{0}^{t} 1_{\partial \Omega}\left(y_{x}(s)\right) \mathrm{d} L_{s}, \text { for } t \geq 0 .
\end{array}\right.
$$

This is one of the simpler ways of realizing state constraints by specific boundary action on the system. Heuristically speaking, this dynamic problem corresponds to a usual controlled ordinary differential equation (as 
for example (1.3)) while $y_{x}(t)$ lies in $\Omega$; but, when $y_{x}(t)$ crosses $\partial \Omega, y_{x}(t)$ is pushed back along the direction $\gamma\left(y_{x}(t)\right)$ with a force $\mathrm{d} L_{t}$. We recall that the above Skorokhod problem admits a unique solution $\left(y_{x}(t), L_{t}\right)$ where $y_{x}(\cdot) \in \mathcal{C}\left(\left[0, \infty[, \bar{\Omega})\right.\right.$ and $L_{t} \in B V(0, T) \forall T<\infty$, where $B V(0, T)$ is the set of bounded variation functions on $[0, T]$ (see for instance [31]).

For both games, constants $\lambda_{0}>0$ and $K \geq 1$ exist such that

$$
\left|y_{x}(t ; a(t), b(t))-y_{z}(t ; a(t), b(t))\right| \leq K \mathrm{e}^{\lambda_{0} t}|x-z|
$$

$\forall x, z \in \bar{\Omega}, a(\cdot) \in \mathcal{A}, b(\cdot) \in \mathcal{B}$ and $t \geq 0$. For the periodic case the proof can be found in [8] or [28], while, here (see appendix), we prove the estimate (3.3) considering the Skorokhod problem given by the ODE (3.2).

The property (3.3) implies that, in both the periodic and the Neumann boundary conditions, we have the following Hölder estimate about the (lower) value function $v_{\lambda}$, defined in (2.4).

Lemma 3.1. For all $\lambda>0$ such that $\lambda<\lambda_{0}$, we have the following estimate

$$
\left|\lambda v_{\lambda}(x)-\lambda v_{\lambda}(z)\right| \leq C|x-z|^{\frac{\lambda}{\lambda_{0}}} \quad \forall x, z \in \bar{\Omega}
$$

where $C>0$ is a constant which does not depend on $\lambda$.

Proof. The proof is classical, but we write it here for the sake of completeness; in particular, we want to point out that the constant $C$ does not depend on $\lambda$ and how using that $\Omega$ is bounded. For any $\varepsilon>0$ there exists $\tilde{\alpha}$ such that

$$
\lambda v_{\lambda}(z)+\lambda \varepsilon \geq \lambda \sup _{b \in \mathcal{B}}\left\{\int_{0}^{T} l\left(y_{z}(t), \tilde{\alpha}[b](t), b(t)\right) \mathrm{e}^{-\lambda t} \mathrm{~d} t+\mathrm{e}^{-\lambda T} v_{\lambda}\left(y_{z}(T ; \tilde{\alpha}[b], b)\right)\right\}
$$

for all $T>0$. Hence, we can choose $\tilde{b} \in \mathcal{B}$ so that

$$
\lambda v_{\lambda}(x)-\lambda v_{\lambda}(z)-2 \lambda \varepsilon \leq \lambda \int_{0}^{T}\left[l\left(y_{x}(t), \tilde{\alpha}[\tilde{b}](t), \tilde{b}(t)\right)-l\left(y_{z}(t), \tilde{\alpha}[\tilde{b}](t), \tilde{b}(t)\right)\right] \mathrm{e}^{-\lambda t} \mathrm{~d} t+2 M \mathrm{e}^{-\lambda T}
$$

because $l$ is uniformly bounded by $M$. The function $l$ is also Lipschitz in $x$, uniformly in $a$ and $b$, therefore, we obtain

$$
\begin{aligned}
\left|\lambda v_{\lambda}(x)-\lambda v_{\lambda}(z)\right| & \leq 2 \lambda \varepsilon+\lambda M \int_{0}^{T}\left|y_{x}(t ; \tilde{\alpha}[\tilde{b}](t), \tilde{b}(t))-y_{z}(t ; \tilde{\alpha}[\tilde{b}](t), \tilde{b}(t))\right| \mathrm{e}^{-\lambda t} \mathrm{~d} t+2 M \mathrm{e}^{-\lambda T} \\
& \leq 2 \lambda \varepsilon+\lambda M K \int_{0}^{T} \mathrm{e}^{\left(\lambda_{0}-\lambda\right) t}|x-z| \mathrm{d} t+2 M \mathrm{e}^{-\lambda T} \\
& \leq 2 \lambda \varepsilon+\frac{\lambda M K}{\lambda_{0}-\lambda}|x-z|\left[\mathrm{e}^{\left(\lambda_{0}-\lambda\right) T}-1\right]+2 M \mathrm{e}^{-\lambda T}
\end{aligned}
$$

where in the second inequality we use the estimate (3.3). Without loss of generality, we can suppose that $|x-z|<\frac{2}{K}$ and take $T_{0}=\frac{1}{\lambda_{0}} \log \left(\frac{2}{K|x-z|}\right)>0$, which minimizes the function

$$
G(T):=\frac{\lambda M K}{\lambda_{0}-\lambda}|x-z|\left[\mathrm{e}^{\left(\lambda_{0}-\lambda\right) T}-1\right]+2 M \mathrm{e}^{-\lambda T},
$$

obtaining

$$
\begin{aligned}
\left|\lambda v_{\lambda}(x)-\lambda v_{\lambda}(z)\right| & \leq 2 \lambda \varepsilon+\frac{\lambda_{0}}{\lambda_{0}-\lambda} M 2^{1-\frac{\lambda}{\lambda_{0}}} K^{\frac{\lambda}{\lambda_{0}}}|x-z|^{\frac{\lambda}{\lambda_{0}}} \\
& \leq 2 \lambda \varepsilon+2 M K|x-z|^{\frac{\lambda}{\lambda_{0}}} .
\end{aligned}
$$

The proof in the periodic case is the same considering $K=1$. 


\subsection{The asymptotic behavior}

In this subsection, our purpose is to prove the uniform convergence of the value function $v_{\lambda}$ (defined in (2.4)) to a constant in the whole $\bar{\Omega}$. This property characterizes the ergodicity in differential games. Therefore, we start introducing the following notion.

Definition 3.2 (ergodic game). The differential game is called ergodic if there exists a (unique) constant real number $\chi_{0}$ such that for all $x \in \bar{\Omega}$, we have

$$
\lim _{\lambda \rightarrow 0} \lambda v_{\lambda}(x)=\chi_{0}
$$

We extend the notion of uniform approximate controllability to differential games; this notion was given by Arisawa in [4] for the (one player) control theory.

Definition 3.3. Let $\Omega$ be a domain in $\mathbb{R}^{n}$. We say that a point $x \in \bar{\Omega}$ is approximately controllable by the first player to a point $z \in \bar{\Omega}$ with the estimate $\delta(\varepsilon ; x, z)$ if for any $\varepsilon>0$ fixed there exists a strategy of the first player, $\tilde{\alpha} \in \Delta$, such that $\forall b(\cdot) \in \mathcal{B} \exists T(\varepsilon ; x, z, \tilde{\alpha}, b(\cdot)) \geq 0$ we have

$$
\left|y_{x}(T(\varepsilon ; x, z, \tilde{\alpha}, b(\cdot)))-z\right|<\varepsilon, \quad T(\varepsilon ; x, z, \tilde{\alpha}, b(\cdot)) \leq \delta(\varepsilon ; x, z),
$$

where it is understood that $y_{x}(T)=y_{x}(T, \tilde{\alpha}[b(\cdot)], b(\cdot))$.

Definition 3.4 (uniform approximate controllability). The (controlled) system (1.3) ((1.4) respectively) has the property of being uniformly approximately controllable by the first player if $\kappa \in[0,1)$ and $N>0$ exist such that any point $x \in \bar{\Omega}$ is approximately controllable to any point $z \in \bar{\Omega}$ with an estimate $\delta(\varepsilon ; x, z)$ such that

$$
\delta(\varepsilon ; x, z) \leq N(-\log \varepsilon)^{\kappa} \quad \forall \varepsilon>0, \forall x, z \in \bar{\Omega}
$$

We recall that we have a Dynamic Programming Principle for the (lower) value $v_{\lambda}$ (see for instance the book [8] and for the Neumann case see [30]): for all $T>0$

$$
v_{\lambda}(x)=\inf _{\alpha \in \Delta} \sup _{b \in \mathcal{B}}\left\{\int_{0}^{T} l\left(y_{x}(t), \alpha[b](t), b(t)\right) \mathrm{e}^{-\lambda t} \mathrm{~d} t+\mathrm{e}^{-\lambda T} v_{\lambda}\left(y_{x}(T ; \tilde{\alpha}[b], b)\right)\right\} .
$$

The following notion will be useful for the proof of the ergodicity result.

Definition 3.5. A functional

$$
\sigma: \mathcal{A} \times \mathcal{B} \longrightarrow[0, \infty]
$$

is nonanticipating if

$$
a(t)=\hat{a}(t), b(t)=\hat{b}(t) \text { for a.e. } 0 \leq t \leq \sigma(a(\cdot), b(\cdot)) \Longrightarrow \sigma(a(\cdot), b(\cdot))=\sigma(\hat{a}(\cdot), \hat{b}(\cdot)) .
$$

Theorem 3.6 (ergodicity for periodic and Neumann boundary conditions). If the system (1.3) ((1.4) respectively) is uniformly approximately controllable (by the first player), then the differential game is ergodic.

Proof. By the uniform approximate controllability for any $x, z \in \bar{\Omega}$ and $\varepsilon>0$ there exists a strategy (of the first player), say $\tilde{\alpha}$, such that $\forall b \in \mathcal{B} \exists T(\varepsilon ; x, z, \tilde{\alpha}, b) \geq 0$ satisfying

$$
\mid y_{x}\left(T(\varepsilon ; x, z, \tilde{\alpha}, b)-z \mid<\varepsilon \text { and } T(\varepsilon ; x, z, \tilde{\alpha}, b) \leq N(-\log \varepsilon)^{\kappa} .\right.
$$

Suppose that $x, z \in \bar{\Omega}$ and $\varepsilon>0$ are fixed, let us define $\tilde{T}(\alpha, b)$ as the minimum $T$ such that the above condition holds. Hence, $\tilde{T}(\alpha, b)$ is a nonanticipating functional and we can apply a generalized version of the Dynamic 
Programming Principle (see Th. 3.1 in [21]). Using the estimate (3.4) of Lemma 3.1 we obtain

$$
\left|\lambda v_{\lambda}\left(y_{x}(\tilde{T})\right)-\lambda v_{\lambda}(z)\right| \leq C\left|y_{x}(\tilde{T})-z\right|^{\frac{\lambda}{\lambda_{0}}} \leq C \varepsilon^{\frac{\lambda}{\lambda_{0}}} .
$$

On the other hand, by (3.5) and using the strategy $\tilde{\alpha}$, we get $($ with $\tilde{T}=\tilde{T}(\tilde{\alpha}, b))$

$$
v_{\lambda}(x) \leq \sup _{b \in \mathcal{B}}\left\{\int_{0}^{\tilde{T}} l\left(y_{x}(t), \tilde{\alpha}[b](t), b(t)\right) \mathrm{e}^{-\lambda t} \mathrm{~d} t+\mathrm{e}^{-\lambda \tilde{T}} v_{\lambda}\left(y_{x}(\tilde{T} ; \tilde{\alpha}[b], b)\right)\right\} .
$$

Hence $\forall \delta>0$ there exists $\tilde{b} \in \mathcal{B}$ such that

$$
v_{\lambda}(x) \leq \int_{0}^{\tilde{T}} l\left(y_{x}(t), \tilde{\alpha}[\tilde{b}](t), \tilde{b}(t)\right) \mathrm{e}^{-\lambda t} \mathrm{~d} t+\mathrm{e}^{-\lambda \tilde{T}} v_{\lambda}\left(y_{x}(\tilde{T} ; \tilde{\alpha}[\tilde{b}], \tilde{b})\right)+\delta .
$$

For $y_{x}(\tilde{T})=y_{x}(\tilde{T} ; \tilde{\alpha}[\tilde{b}], \tilde{b})$ we obtain

$$
v_{\lambda}(x)-v_{\lambda}\left(y_{x}(\tilde{T})\right) \leq \int_{0}^{\tilde{T}} l\left(y_{x}(t), \tilde{\alpha}[\tilde{b}](t), \tilde{b}(t)\right) \mathrm{e}^{-\lambda t} \mathrm{~d} t+\left(\mathrm{e}^{-\lambda \tilde{T}}-1\right) v_{\lambda}\left(y_{x}(\tilde{T})\right)+\delta
$$

and, hence,

$$
\left|\lambda v_{\lambda}(x)-\lambda v_{\lambda}\left(y_{x}(\tilde{T})\right)\right| \leq 2 M\left(1-\mathrm{e}^{-\lambda \tilde{T}}\right)+\lambda \delta
$$

where $\tilde{T} \leq N(-\log \varepsilon)^{\kappa}(\kappa \in[0,1))$.

By (3.6) and (3.7) we conclude with the following estimates

$$
\begin{aligned}
\left|\lambda v_{\lambda}(x)-\lambda v_{\lambda}(z)\right| & \leq\left|\lambda v_{\lambda}(x)-\lambda v_{\lambda}\left(y_{x}(\tilde{T})\right)\right|+\left|\lambda v_{\lambda}\left(y_{x}(\tilde{T})\right)-\lambda v_{\lambda}(z)\right| \\
& \leq \lambda \delta+C\left(1-\mathrm{e}^{-\lambda \tilde{T}}+\varepsilon^{\frac{\lambda}{\lambda_{0}}}\right)
\end{aligned}
$$

$\left(C \geq 2 M\right.$ : see the proof of Lem. 3.1). Now, choosing $\varepsilon=\exp \left(-\lambda^{-(1+\omega)}\right)$ with $0<\omega<\frac{1}{\kappa}-1$, the last term on the right hand side of (3.8) goes to zero as $\lambda \rightarrow 0^{+}$. Therefore, for some subsequence $\lambda_{0} \stackrel{\kappa}{\rightarrow} 0^{+}$of $\lambda \rightarrow 0^{+}$, there exists a constant $\chi_{0}$ such that

$$
\lim _{\lambda_{0} \rightarrow 0^{+}} \lambda_{0} v_{\lambda_{0}}(x)=\chi_{0} \quad \text { uniformly in } x \in \bar{\Omega} .
$$

In order to prove the uniqueness of the constant $\chi_{0}$, we use a standard argument based on the Comparison Principle $(c f .[4,30]$ or $[8])$. Assume that $\lim _{\lambda_{1} \rightarrow 0^{+}} \lambda_{1} v_{\lambda_{1}}(x)=\chi_{1}$ uniformly in $x \in \bar{\Omega}$, where $\chi_{1} \neq \chi_{0}$ and $\lambda_{1} \rightarrow 0^{+}$is another subsequence of $\lambda \rightarrow 0^{+}$. We can suppose that $\chi_{0}<\chi_{1}$, for instance. Now, take $v_{0}$ and $v_{1}$ viscosity solutions of $H(x, D u)+\chi_{0}=0$ and $H(x, D u)+\chi_{1}=0$, respectively; we can assume $v_{0}<v_{1}$ because $v_{0}$ and $v_{1}$ are defined up to a constant. Then, choose $\epsilon>0$ sufficiently small so that

$$
\chi_{1}-\epsilon v_{1}>\chi_{0}-\epsilon v_{0} \quad \text { on } \bar{\Omega} .
$$

Since $v_{0}$ is a supersolution of $H(x, D u)+\chi_{0}=0$, then it is also supersolution of

$$
H(x, D u)+\chi_{1}+\epsilon v_{0}-\epsilon v_{1}=0 \text { in } \Omega
$$

with the corresponding boundary conditions (periodic or Neumann type). Now, $v_{1}$ is a viscosity subsolution of (3.9). Thus, thanks to Comparison Theorems (see [28] or [8] for periodic case and [30] for Neumann B.C.) we get $v_{1} \leq v_{0}$ that is a contradiction. So, we get $\chi_{0}=\chi_{1}$. 
Remark 3.7. Instead of considering in the HJI equation (1.1) the so-called "lower Hamiltonian" $H$, defined in (2.6), with the corresponding lower value $v_{\lambda}(x)$, defined in (2.4), one can consider the upper Hamiltonian $\tilde{H}$, given by (2.7) and the upper value function $u_{\lambda}(x)$ (see (2.5)). Therefore, we obtain the same result of ergodicity. In the latter case, we must change the definition of uniform approximate controllability of the first player by just switching controls and strategies: we should say that a point $x \in \bar{\Omega}$ is approximately controllable by the first player to a point $z \in \bar{\Omega}$ with the estimate $\delta(\varepsilon ; x, z)$ if for any $\varepsilon>0$ fixed there exists a control of the first player, $\tilde{a}(\cdot) \in \mathcal{A}$, such that for all strategies $\beta \in \Gamma \exists T(\varepsilon ; x, z, \tilde{a}(\cdot), \beta) \geq 0$ we have

$$
\left|y_{x}(T(\varepsilon ; x, z, \tilde{a}(\cdot), \beta))-z\right|<\varepsilon, \quad T(\varepsilon ; x, z, \tilde{a}(\cdot), \beta) \leq \delta(\varepsilon ; x, z) .
$$

\section{The state constraints B.C.}

In this section, the state space is still the closure of a smooth bounded connected domain $\Omega \subset \mathbb{R}^{n}$ (as in Neumann B.C.); but, in state constraints boundary conditions, we have to face quite different difficulties. Actually, in all the results of the first two Subsections, we do not need that $\Omega$ is bounded or connected. Recall that, in the state constraints case, the ODE is (1.5). Let us start with some basic assumptions and preliminary results.

\subsection{Preliminaries: an example of state constrained game}

We suppose that $A, B \subset \mathbb{R}^{m}$ are compact sets and that $A$ is convex. Moreover, we assume that the set $\bar{\Omega}$ is described by a function $\phi \in \mathcal{C}^{2}\left(\mathbb{R}^{n}, \mathbb{R}\right)$

$$
\bar{\Omega}=\left\{x \in \mathbb{R}^{n} \mid \phi(x) \leq 0\right\} \text { with } \nabla \phi(x) \neq 0 \text { for any } x \in \partial \Omega .
$$

Here, the dynamic is given by the function $f$ so defined:

$$
f(x, a, b)=f_{1}(x) a+f_{2}(x, b)
$$

where $f_{1}$ is an invertible matrix such that $\left\|f_{1}\right\|_{\infty},\left\|f_{1}^{-1}\right\|_{\infty} \leq M_{1}$ for a suitable positive constant $M_{1}$. We recall the reader that $M>0$ is the Lipschitz constant of the function $f$ and we have $\|f(x, a, b)\|_{\infty} \leq M$, too. So, for any starting point $x \in \bar{\Omega}$ and for any couple of controls $(a(\cdot), b(\cdot)) \in \mathcal{A} \times \mathcal{B}$ the system we consider is given by

$$
\left\{\begin{array}{l}
\dot{y}(t)=f_{1}(y(t)) a(t)+f_{2}(y(t), b(t)) \\
y(0)=x \\
y(t) \in \bar{\Omega}
\end{array}\right.
$$

We also have to introduce the notions of admissible controls and strategies; for any $x \in \bar{\Omega}$ we define:

$$
\begin{aligned}
& \mathcal{A D}(x):=\left\{(a(\cdot), b(\cdot)) \in \mathcal{A} \times \mathcal{B} \mid y_{x}(t ; a(t), b(t)) \in \bar{\Omega} \forall t \geq 0\right\} \\
& \mathcal{A}(x):=\{a(\cdot) \in \mathcal{A} \mid \exists b(\cdot) \in \mathcal{B} \text { s. t. }(a(\cdot), b(\cdot)) \in \mathcal{A D}(x)\} \\
& \mathcal{B}(x):=\{b(\cdot) \in \mathcal{B} \mid \exists a(\cdot) \in \mathcal{A} \text { s. t. }(a(\cdot), b(\cdot)) \in \mathcal{A D}(x)\} \\
& \Delta(x):=\{\alpha \in \Delta \mid(\alpha[b], b) \in \mathcal{A D}(x) \forall b(\cdot) \in \mathcal{B}(x)\} .
\end{aligned}
$$

Moreover, we need a viability condition:

$$
\exists \eta>0 \text { such that } \forall x \in \partial \Omega, b \in B \text { there exists } a_{x, b} \in A \text { with }\left\langle f\left(x, a_{x, b}, b\right), \nabla \phi(x)\right\rangle<-\eta<0 .
$$




\section{Remark 4.1.}

a) If $\bar{\Omega}$ is bounded, then (4.3) is equivalent to

$$
\exists \eta>0 \text { such that } \forall x \in \partial \Omega, b \in B \text { there exists } a_{x, b} \in A \text { with }\left\langle f\left(x, a_{x, b}, b\right), \frac{\nabla \phi(x)}{|\nabla \phi(x)|}\right\rangle<-\eta<0 .
$$

But, in any case (even if $\bar{\Omega}$ is not bounded), once we fix a control $b(\cdot) \in \mathcal{B}$, we define the set-valued map

$$
(t, x) \mapsto F_{b}(t, x):=f(x, A, b(t))=\cup_{a \in A} f(x, a, b(t)) .
$$

Notice that under the above condition (4.3), since we always get a control $a_{x, b(t)} \in A$ such that

$$
\left\langle f\left(x, a_{x, b(t)}, b(t)\right), \frac{\nabla \phi(x)}{|\nabla \phi(x)|}\right\rangle \leq 0 \quad \forall x \in \partial \Omega,
$$

by applying the Measurable Viability Theorem of [23], there exists a solution to the problem

$$
\left\{\begin{array}{l}
\dot{y}(t) \in F_{b}(t, y(t)) \\
y(0)=x \\
y(t) \in \bar{\Omega}
\end{array}\right.
$$

Therefore, the hypothesis (4.3) implies that $\forall x \in \bar{\Omega} \mathcal{B}(x) \neq \emptyset$ (in fact $\mathcal{B}(x)=\mathcal{B}$ ) or, equivalently, $\mathcal{A}(x) \neq \emptyset$.

b) In what follows, we will show how to construct not only admissible controls for the first player, but also "good" strategies, which play a crucial role in proving regularity results on the value function.

In this example, for any starting point $x \in \bar{\Omega}$ and $\forall(a(\cdot), b(\cdot)) \in \mathcal{A D}(x)$ the cost functional is the same as before:

$$
J_{\lambda}^{T}(x, a(\cdot), b(\cdot))=\int_{0}^{T} l\left(y_{x}(t ; a(t), b(t)), a(t), b(t)\right) \mathrm{e}^{-\lambda t} \mathrm{~d} t,
$$

where the Lagrangian $l: \mathbb{R}^{n} \times A \times B \longrightarrow \mathbb{R}$ is bounded and Lipschitz continuous (of constant $M$ ).

Let us define the (lower) value function as follows ( $c f .[27])$ :

$$
V_{\lambda}(x)=\inf _{\alpha \in \Delta(x)} \sup _{b \in \mathcal{B}(x)} J_{\lambda}^{\infty}(x, \alpha[b], b) .
$$

If $K \subset \mathbb{R}^{n}$ is a closed convex set, we denote the projection map on $K$ by $\pi_{K}: \mathbb{R}^{n} \mapsto K ; \pi_{K}$ is well defined (see for example [7]). Now, suppose that the couple of controls $\left(a_{1}(\cdot), b_{1}(\cdot)\right) \in \mathcal{A} \times \mathcal{B}$ is admissible for the point $x_{1} \in \bar{\Omega}$; then, we consider the system:

$$
\left\{\begin{array}{l}
\dot{y}(t)=\pi_{F_{b_{1}}(t, y(t)) \cap T_{\bar{\Omega}}(y(t))}\left(f\left(y(t), a_{1}(t), b_{1}(t)\right)\right) \\
y(0)=x_{2} \in \bar{\Omega}
\end{array}\right.
$$

where we recall that $F_{b_{1}}(t, y):=f\left(y, A, b_{1}(t)\right)\left(c f\right.$. Rem. 4.1), $T_{\bar{\Omega}}(x)$ is the usual tangent half-space at $x$ to the set $\bar{\Omega}$ with a smooth boundary and $x_{2}\left(x_{2} \neq x_{1}\right)$ is a new starting point.

In the system (4.5), roughly speaking, whenever the controls $a_{1}(\cdot)$ and $b_{1}(\cdot)$ do not push outside to $\bar{\Omega}$ the trajectory starting from $x_{2}$, the first player continues to use the control $a_{1}(\cdot)$. Otherwise, he chooses a control such that the velocity of the trajectory is precisely the unique projection of the vector field $f\left(y(\cdot), a_{1}(\cdot), b_{1}(\cdot)\right)$ on the closed convex set $F_{b_{1}}(\cdot, y(\cdot)) \cap T_{\bar{\Omega}}(y(\cdot))$. This happens, for example, when the trajectory $y_{x_{2}}(t)$ is on $\partial \Omega$ at time $t$ and

$$
\left\langle f\left(y(t), a_{1}(t), b_{1}(t)\right), \nabla \phi(y(t))\right\rangle>0 .
$$


Thanks to the injectivity of $f_{1}$, there exists only one control $\bar{a}(t, x) \in A$ such that

$$
f\left(x, \bar{a}(t, x), b_{1}(t)\right)=\pi_{F_{b_{1}}(t, x) \cap T_{\bar{\Omega}}(x)}\left(f\left(x, a_{1}(t), b_{1}(t)\right)\right) .
$$

In the following four lemmas, we just adapt some arguments developed in [12] to our case; we recall that, in the article [12], the dynamics are given by a function of the following kind: $f(x, a, b)=\left\{g\left(x_{1}, a\right)\right\} \times\left\{h\left(x_{2}, b\right)\right\}$ where $\left(x_{1}, x_{2}\right)=x, x_{1} \in \mathbb{R}^{n_{1}}$ and $x_{2} \in \mathbb{R}^{n_{2}}$ with $n_{1}+n_{2}=n$. Actually, what we need is to extend the approach of [12] to vector fields depending also on time. Here, we write the proofs with essential modifications in short. We refer the reader to [12] for further details.

Lemma 4.2. Assume (4.1) and (4.3). Then, for any starting point $x \in \bar{\Omega}$ system (4.5) admits solutions.

Proof. System (4.5) is equivalent to system

$$
\left\{\begin{array}{l}
\dot{y}(t) \in \tilde{F}(t, y(t)) \\
y(t) \in \bar{\Omega} \\
y(0)=x_{2} \in \bar{\Omega}
\end{array}\right.
$$

where

$$
\tilde{F}(t, x):=\left\{\begin{array}{l}
f\left(x, a_{1}(t), b_{1}(t)\right) \text { if } x \in \operatorname{Int}(\Omega) \\
\overline{\operatorname{co}}\left\{f\left(x, a_{1}(t), b_{1}(t)\right) ; f\left(x, \bar{a}(t, x), b_{1}(t)\right)\right\} \text { if } y \in \partial \Omega
\end{array}\right.
$$

(here, if $Y \subset \mathbb{R}^{n}$, then $\overline{c o} Y$ denotes the closed convex hull of $Y$ ). The set-valued function $(t, x) \mapsto \tilde{F}(t, x)$ is upper semicontinuous with respect to $x$ and measurable with respect to $t$. Therefore, by the Measurable Viability theorem of [23], we obtain that system (4.6) admits solutions for any starting point $x_{2} \in \bar{\Omega}$.

An immediate consequence is the following corollary.

Corollary 4.3. For any $x \in \bar{\Omega}$ we have $\mathcal{B}(x) \equiv \mathcal{B}$ and $\forall b(\cdot) \in \mathcal{B}$ we get $(a(\cdot), b(\cdot)) \in \mathcal{A D}(x)$ by choosing a $(\cdot)$ such that

$$
f(x, a(t), b(t))=\pi_{F_{b}(t, x) \cap T_{\bar{\Omega}}(x)}\left(f\left(x, a_{1}(t), b(t)\right)\right) .
$$

Proof. Take $b(\cdot) \in \mathcal{B}$. For all $x \in \bar{\Omega}$, consider system (4.5) with $b_{1}(\cdot)=b(\cdot), x_{2}=x$ and any $a_{1}(\cdot) \in \mathcal{A}$. Then, system (4.5) admits solutions and, by choosing the control $a(\cdot)$ that realizes the projection in (4.5), permits us to get also an admissible couple of controls $(a(\cdot), b(\cdot)) \in \mathcal{A D}(x)$.

The following lemma provides the crucial estimate on controls.

Lemma 4.4. Under assumptions (4.1) and (4.3), there exists a positive constant $\hat{C}$ such that for any $x_{1}, x_{2} \in \bar{\Omega}$, for any admissible couple of admissible controls $\left(a_{1}(\cdot), b_{1}(\cdot)\right) \in \mathcal{A D}\left(x_{1}\right)$, it is possible to find an admissible control $a_{2}(\cdot) \in \mathcal{A}\left(x_{2}\right)$, which is admissible with the same control $b_{1}(\cdot) \in \mathcal{B}\left(x_{1}\right)$ and

$$
\int_{0}^{t}\left|a_{1}(s)-a_{2}(s)\right| \mathrm{d} s \leq\left(\left|x_{1}-x_{2}\right|+\int_{0}^{t}\left|y_{x_{1}}\left(t ; a_{1}, b_{1}\right)(s)-y_{x_{2}}\left(t ; a_{2}, b_{1}\right)(s)\right| \mathrm{d} s\right) .
$$

The long proof is exactly as in [12], to which we refer the reader; we only underline the fact that $a_{2}(\cdot)$ is so that it realizes the projection in system (4.5).

Lemma 4.5. Assume that (4.1) and (4.3) hold true. Then, there exists some positive $K_{0}$ such that for any $x_{1}, x_{2} \in \bar{\Omega}$, for any couple of admissible controls $\left(a_{1}(\cdot), b_{1}(\cdot)\right) \in \mathcal{A D}\left(x_{1}\right)$, it is possible to find a control $a_{2}(\cdot) \in$ $\mathcal{A}\left(x_{2}\right)$, which is admissible with the same control $b_{1}(\cdot) \in \mathcal{B}\left(x_{1}\right)$, such that $\forall t \geq 0$ we have

$$
\left|y_{x_{1}}\left(t ; a_{1}, b_{1}\right)-y_{x_{2}}\left(t ; a_{2}, b_{1}\right)\right|+\int_{0}^{t}\left|a_{1}(s)-a_{2}(s)\right| \mathrm{d} s \leq K_{0}\left|x_{1}-x_{2}\right| \mathrm{e}^{K_{0} t} .
$$


Proof. By the construction of the trajectories, we get

$$
\begin{aligned}
\left|y_{x_{1}}\left(t ; a_{1}, b_{1}\right)-y_{x_{2}}\left(t ; a_{2}, b_{1}\right)\right| & \leq\left|x_{1}-x_{2}\right|+\int_{0}^{t}\left|f\left(y_{x_{1}}(s), a_{1}(s), b_{1}(s)\right)-f\left(y_{x_{2}}(s), a_{2}(s), b_{1}(s)\right)\right| \mathrm{d} s \\
& \leq\left|x_{1}-x_{2}\right|+M \int_{0}^{t}\left|a_{1}(s)-a_{2}(s)\right| \mathrm{d} s+M\left(C_{A}+C_{B}+1\right) \int_{0}^{t}\left|y_{x_{1}}(s)-y_{x_{2}}(s)\right| \mathrm{d} s
\end{aligned}
$$

where $C_{A}:=\max _{a \in A}|a|$ and $C_{B}:=\max _{b \in B}|b|$.

But, using the previous Lemma 4.5 and a suitable selection argument, we obtain the same estimate also by suitably choosing admissible strategies for the first player.

Lemma 4.6. Assume (4.1) and (4.3). Then, there exist a positive real number $K_{0}$ such that for any $x_{1}, x_{2} \in \bar{\Omega}$ and $\forall t \geq 0$ and for any strategy $\alpha_{1} \in \Delta\left(x_{1}\right)$, there is a nonanticipative strategy $\alpha_{2}: \mathcal{B}\left(x_{2}\right) \longrightarrow \mathcal{A}\left(x_{2}\right)$ with the following property: for any $b(\cdot) \in \mathcal{B}\left(x_{2}\right)$ we have

$$
\left|y_{x_{1}}\left(t ; \alpha_{1}[b], b\right)-y_{x_{2}}\left(t ; \alpha_{2}[b], b\right)\right|+\int_{0}^{t}\left|\alpha_{1}[b](s)-\alpha_{2}[b](s)\right| \mathrm{d} s \leq K_{0}\left|x_{1}-x_{2}\right| \mathrm{e}^{K_{0} t}
$$

Proof. For any $\left(a_{1}(\cdot), b(\cdot)\right) \in \mathcal{A D}\left(x_{1}\right)$, we can find a control $a_{2}(\cdot) \in \mathcal{A}\left(x_{2}\right)$ such that the following system is satisfied

$$
\left\{\begin{array}{l}
\dot{y}(t)=f(y(t), a(t), b(t))=\pi_{F_{b}(t, y(t)) \cap T_{\bar{\Omega}}(y(t))}\left(f\left(y(t), a_{1}(t), b(t)\right)\right) \\
y(0)=x_{2} \in \bar{\Omega} .
\end{array}\right.
$$

Let us consider the set-valued map $\Sigma: \mathcal{A D}\left(x_{1}\right) \longrightarrow \mathcal{A}\left(x_{2}\right)$ defined by:

$$
\Sigma\left(a_{1}(\cdot), b(\cdot)\right):=\left\{a_{2}(\cdot) \in \mathcal{A}\left(x_{2}\right) \mid(4.7) \text { is satisfied }\right\}
$$

This set-valued map is nonexpansive with nonempty $(*)$-closed values (in the sense of [15]), and, hence, by the Plaskacz Lemma (see Lem. 2.7 of [15]) it turns out that there exists a nonanticipative selection $\alpha$ with the following property: $\alpha\left(a_{1}(\cdot), b(\cdot)\right) \in \Sigma\left(a_{1}(\cdot), b(\cdot)\right)$ for any $\left(a_{1}(\cdot), b(\cdot)\right) \in \mathcal{A D}\left(x_{1}\right)$. We recall that $\forall b(\cdot) \in \mathcal{B}$ there exists a control $a_{1}(\cdot) \in \mathcal{A}\left(x_{1}\right)$ such that $\left(a_{1}(\cdot), b(\cdot)\right) \in \mathcal{A D}\left(x_{1}\right)$ (see Rem. 4.1 and Cor. 4.3). For all $b(\cdot) \in \mathcal{B}$ we set $\alpha_{2}[b]:=\alpha\left(\alpha_{1}[b], b\right)$. Notice that $\alpha_{2}$ belongs to $\Delta\left(x_{2}\right)$ since $\alpha_{1}$ and $\alpha$ are nonanticipative: indeed, if $b_{1}(\cdot)=b_{2}(\cdot)$ on $[0, t]$ then $\alpha_{1}\left[b_{1}(\cdot)\right]=\alpha_{1}\left[b_{2}(\cdot)\right]$ on $[0, t]$ and so $\alpha_{2}\left[b_{1}(\cdot)\right]=\alpha_{2}\left[b_{2}(\cdot)\right]$ on $[0, t]$.

Finally, we just apply the previous Lemma 4.5 with $a_{1}(\cdot)=\alpha_{1}[b(\cdot)](\cdot)$ and $a_{2}(\cdot)=\alpha_{2}[b(\cdot)](\cdot)$.

\subsection{Regularity of the value function}

We recall that for the (lower) value function $V_{\lambda}$, defined in (4.4), the Dynamic Programming Principle holds. The statement, we use, is a slightly different version of the Koike's one in [27]: we need to adapt it to our problem. The proof is quite the same (see, for instance, [11]).

Lemma 4.7. For any $x \in \bar{\Omega}$ and $T>0$, we have

$$
V_{\lambda}(x)=\inf _{\alpha \in \Delta(x)} \sup _{b \in \mathcal{B}}\left\{\int_{0}^{T} l\left(y_{x}(t ; \alpha[b](t), b(t))\right) \mathrm{e}^{-\lambda t} \mathrm{~d} t+\mathrm{e}^{-\lambda T} V_{\lambda}\left(y_{x}(T ; \alpha[b], b)\right)\right\}
$$

We get an Hölder estimate of the term $\lambda V_{\lambda}(x)$ similarly as in Lemma 3.1; however, here, we have to be careful in the choice of the admissible strategies and controls.. 
Proposition 4.8 (Hölder continuity). Assume (4.1) and (4.3). For any $\lambda>0, \lambda<K_{0}$, we have the following estimate

$$
\left|\lambda V_{\lambda}\left(x_{1}\right)-\lambda V_{\lambda}\left(x_{2}\right)\right| \leq \tilde{M}\left|x_{1}-x_{2}\right|^{\frac{\lambda}{k_{0}}} \quad \forall x_{1}, x_{2} \in \bar{\Omega}
$$

where the constant $\tilde{M}>0$ does not depend on $\lambda$.

Proof. By the Dynamic Programming Principle (Lem. 4.7) we have that $\forall T>0 \forall x_{1} \in \bar{\Omega}$ :

$$
V_{\lambda}\left(x_{1}\right)=\inf _{\alpha \in \Delta\left(x_{1}\right)} \sup _{b \in \mathcal{B}}\left\{\int_{0}^{T} l\left(y_{x_{1}}(t ; \alpha[b](t), b(t))\right) \mathrm{e}^{-\lambda t} \mathrm{~d} t+\mathrm{e}^{-\lambda T} V_{\lambda}\left(y_{x_{1}}(T ; \alpha[b], b)\right)\right\} .
$$

Hence for any $\epsilon>0$ there exists a strategy $\alpha_{1}: \mathcal{B}\left(x_{1}\right) \longrightarrow \mathcal{A}\left(x_{1}\right)$ such that

$$
V_{\lambda}\left(x_{1}\right)+\epsilon \geq \sup _{b \in \mathcal{B}}\left\{\int_{0}^{T} l\left(y_{x_{1}}\left(t ; \alpha_{1}[b](t), b(t)\right)\right) \mathrm{e}^{-\lambda t} \mathrm{~d} t+\mathrm{e}^{-\lambda T} V_{\lambda}\left(y_{x_{1}}\left(T ; \alpha_{1}[b], b\right)\right)\right\} .
$$

By applying the strategy $\alpha_{2}: \mathcal{B}\left(x_{2}\right)=\mathcal{B} \longrightarrow \mathcal{A}\left(x_{2}\right)$ of Lemma 4.6 we have

$$
V_{\lambda}\left(x_{2}\right) \leq \sup _{b \in \mathcal{B}}\left\{\int_{0}^{T} l\left(y_{x_{2}}\left(t ; \alpha_{2}[b](t), b(t)\right)\right) \mathrm{e}^{-\lambda t} \mathrm{~d} t+\mathrm{e}^{-\lambda T} V_{\lambda}\left(y_{x_{2}}\left(T ; \alpha_{2}[b], b\right)\right)\right\}
$$

and $\forall \epsilon>0 \exists b_{2}(\cdot) \in \mathcal{B}$ such that

$$
V_{\lambda}\left(x_{2}\right)-\epsilon \leq\left\{\int_{0}^{T} l\left(y_{x_{2}}\left(t ; \alpha_{2}\left[b_{2}\right](t), b_{2}(t)\right)\right) \mathrm{e}^{-\lambda t} \mathrm{~d} t+\mathrm{e}^{-\lambda T} V_{\lambda}\left(y_{x_{2}}\left(T ; \alpha_{2}\left[b_{2}\right], b_{2}\right)\right)\right\} .
$$

On the other hand, using $b_{2}(\cdot) \in \mathcal{B}$ in $(4.10)$, we have

$$
V_{\lambda}\left(x_{1}\right)+\epsilon \geq \int_{0}^{T} l\left(y_{x_{1}}\left(t ; \alpha_{1}\left[b_{2}\right](t), b_{2}(t)\right)\right) \mathrm{e}^{-\lambda t} \mathrm{~d} t+\mathrm{e}^{-\lambda T} V_{\lambda}\left(y_{x_{1}}\left(T ; \alpha_{1}\left[b_{2}\right], b_{2}\right)\right) .
$$

Finally, we obtain

$$
\lambda V_{\lambda}\left(x_{2}\right)-\lambda V_{\lambda}\left(x_{1}\right)-2 \lambda \varepsilon \leq \lambda \int_{0}^{T}\left[l\left(y_{x_{2}}\left(t ; \alpha_{2}\left[b_{2}\right](t), b_{2}(t)\right)\right)-l\left(y_{x_{1}}\left(t ; \alpha_{1}\left[b_{2}\right](t), b_{2}(t)\right)\right] \mathrm{e}^{-\lambda t} \mathrm{~d} t+2 M \mathrm{e}^{-\lambda T}\right.
$$

and, so

$$
\lambda V_{\lambda}\left(x_{2}\right)-\lambda V_{\lambda}\left(x_{1}\right) \leq 2 \lambda \varepsilon+\lambda M K_{0} \mathrm{e}^{K_{0} T} \frac{1+K_{0}}{K_{0}-\lambda}\left|x_{2}-x_{1}\right|+2 M \mathrm{e}^{-\lambda T} .
$$

The conclusion of the proof is exactly as in Lemma 3.1.

\subsection{The asymptotic behavior}

In this section, treating a state constrained problem, we have to be more precise with the concept of approximate controllability by specifying the choice of the strategy of the first player; moreover, we consider a stronger condition than before: the time $T(\cdot)$ does not depend on the control $b(\cdot)$.

Finally, we recall that in this subsection $\bar{\Omega}$ is a bounded connected subset of $\mathbb{R}^{n}$ with property (4.1).

Definition 4.9 (approximate controllability with state constraints). A point $x \in \bar{\Omega}$ is strong approximately controllable by the first player to a point $z \in \bar{\Omega}$ with the estimate $\delta(\varepsilon ; x, z)$, if for any $\varepsilon>0$ fixed there exists a strategy of the first player, $\tilde{\alpha} \in \Delta(x)$, and a time $T(\varepsilon ; x, z, \tilde{\alpha}) \geq 0$ such that $\forall b(\cdot) \in \mathcal{B}(x)$ we have

$$
\left|y_{x}(T(\varepsilon ; x, z, \tilde{\alpha}))-z\right|<\varepsilon, \quad T(\varepsilon ; x, z, \tilde{\alpha}) \leq \delta(\varepsilon ; x, z) .
$$


For any $(x, b) \in \bar{\Omega} \times B$ we define the following subsets:

$$
A(x, b):=\left\{a \in A \mid \exists r>0 \text { s.t. } y_{z}(t, a, b) \in \bar{\Omega} \text { for } t \in[0, r] \text { and } z \in \bar{\Omega} \cap B(x, r)\right\}
$$

and

$$
F_{-}(x, b):=\operatorname{co}\{f(x, a, b) \mid a \in A(x, b)\},
$$

namely, $F_{-}(x, b)$ is the convex hull of the set $f(x, A(x, b), b)$.

Let us introduce a new assumption $(c f .[27])$ :

$$
\left\{\begin{array}{l}
\text { There are } r, s>0 \text { such that, if } \hat{b} \in B \text { and } b(\cdot) \in \mathcal{B} \text { satisfy } \\
|b(t)-\hat{b}|<r \text { a.e. on }[0, s] \text {, and } x \in \partial \Omega \text {, then } \\
A(x, \hat{b}) \neq \emptyset \text { and } y_{x}(t, a, b(\cdot)) \in \bar{\Omega} \text { for } t \in[0, s] \text { and } a \in A(x, \hat{b}) .
\end{array}\right.
$$

Notice that under conditions (4.1) and (4.3) of Section 4.1 we get $a_{x, b} \in A(x, b)$ and, therefore, $A(x, b) \neq \emptyset$ for all $(x, b) \in \bar{\Omega} \times B$.

Suppose that condition (4.11) holds true, then the (lower) value function $V_{\lambda}$ defined in (4.4) is a viscosity solution to the following HJI equation ( $c f .[27])$

$$
\lambda u_{\lambda}+\mathcal{H}\left(x, D u_{\lambda}\right)=0
$$

where

$$
\mathcal{H}(x, p):=\min _{b \in B} \max _{a \in A(x, b)}\{-f(x, a, b) \cdot p-l(x, a, b)\}
$$

Moreover, if also the following assumption holds true

$$
\inf _{x \partial \Omega, b \in B}\left\{\xi \mid \xi \in F_{-}(x, b)\right\}>0
$$

then we obtain a Comparison Theorem (see [27]), thus $V_{\lambda}$ is the unique viscosity solution of (4.12). Since by Proposition 4.7 the (lower) value function $V_{\lambda}$ is such that $\forall x, z \in \bar{\Omega}$

$$
\left|\lambda V_{\lambda}(x)-\lambda V_{\lambda}(z)\right| \leq M^{*}|x-z|^{\frac{\lambda}{\lambda^{*}}}
$$

for some positive constants $\lambda^{*}$ and $M^{*}$, then, analogously to Theorem 3.6, we get an ergodicity result.

Theorem 4.10 (ergodicity for state constraints). Suppose that (4.1), (4.3), (4.11) and (4.13) hold true and that the system (4.2) is strong uniformly approximately controllable by the first player. Then, the differential game is ergodic; namely, $\lambda V_{\lambda}(x)$ converges uniformly to a (unique) constant as $\lambda \rightarrow 0^{+}$.

\section{The Abelian-Tauberian Problem}

Finally, we point out the fact that it is possible to obtain a result that gives equivalent characterizations of the notion of ergodicity. It was proved by [6] for the optimal control problem by using the Dynamic Programming Principle ( $c f$. also [4,8]). For the Neumann B.C., in particular, we refer to the article of P.L. Lions [30]. Successively, in [2] (see also [1]) Alvarez and Bardi give a proof which is valid in the periodic case for an arbitrary Hamiltonian only using the comparison principle and the viscosity solutions theory.

In the following proposition, we simply show that the same arguments (with some adaptations) of the authors above can be used also for the Neumann boundary conditions. We recall that the function $v_{\lambda}(x)$ denotes the viscosity solution of the problem

$$
\left\{\begin{aligned}
\lambda v_{\lambda}+H\left(x, D v_{\lambda}\right) & =0 & & \text { in } \Omega \\
\frac{D v}{D \gamma} & =0 & & \text { on } \partial \Omega
\end{aligned}\right.
$$


Let $v(x, t)$ be the viscosity solution of time dependent problem with Neumann B.C.:

$$
\left\{\begin{aligned}
v_{t}+H(x, D v) & =0 & & \text { in } \Omega \times(0,+\infty) \\
v(x, 0) & =0 & & \text { in } \bar{\Omega} \\
\frac{D v}{D \gamma} & =0 & & \text { on } \Omega \times(0,+\infty),
\end{aligned}\right.
$$

and let $u(x)$ be the viscosity solution of the cell problem for a constant $\chi \in \mathbb{R}$ :

$$
\left\{\begin{aligned}
\chi+H(x, D u) & =0 & & \text { in } \Omega \\
\frac{D u}{D \gamma} & =0 & & \text { on } \partial \Omega .
\end{aligned}\right.
$$

Recall that in our case the Hamiltonian is given by $H(x, p):=\min _{b \in B} \max _{a \in A}\{-f(x, a, b) \cdot p-l(x, a, b)\}$.

Proposition 5.1. Let us suppose that one of the following conditions holds

a) $\lambda v_{\lambda}(x) \longrightarrow \chi_{0}$ as $\lambda \longrightarrow 0^{+}$uniformly in $x$;

b) $\frac{1}{t} v(x, t) \longrightarrow \chi_{1}$ as $t \longrightarrow+\infty$ uniformly in $x$;

c) $\sup \{\chi \mid \exists u$ subsolution of (5.3) $\}=\inf \{\chi \mid \exists$ u supersolution of (5.3) $\}$.

Then, we have that also the other assertions are true and, moreover,

$$
\chi_{0}=\chi_{1}=\chi_{2}
$$

where $\chi_{2}$ is the constant defined by $c$ ).

Remark 5.2. Let us denote by $\chi_{I}$ and $\chi_{S}$ the infimum and supremum in $c$ ) of Proposition 5.1, respectively: namely,

$$
\begin{aligned}
& \chi_{I}:=\inf \{\chi \mid \exists u \text { supersolution of (5.3) }\} \\
& \chi_{S}:=\sup \{\chi \mid \exists u \text { subsolution of (5.3)\}. }
\end{aligned}
$$

We claim that $\chi_{S} \leq \chi_{I}$ (we use the argument of [2]). Indeed, suppose that there exists two real constant $\mu_{0}, \mu_{1}$ and two functions $v_{0}, v_{1} \in \mathcal{C}(\bar{\Omega})$ such that $\mu_{0}<\mu_{1}, v_{1}$ is a subsolution of (5.3) corresponding to $\mu_{1}$ and $v_{0}$ is a supersolution of (5.3) corresponding to $\mu_{0}$. Since $v_{0}$ and $v_{1}$ are defined up to a constant and $\bar{\Omega}$ is a compact set, then we may assume that $v_{0}<v_{1}$. By applying the same comparison argument of Theorem 3.6 (see the part of the proof dealing with the uniqueness of the constant $\chi_{0}$ ), we get that $v_{1} \leq v_{0}$, reaching a contradiction. Thus, we have $\chi_{S} \leq \chi_{I}$.

Proof of Proposition 5.1. $a) \Rightarrow b$ ). Here we use the representation formula of the viscosity solutions of (5.1) and (5.2) (see [30] and $c f .[4]$ ):

$$
v_{\lambda}(x):=\inf _{\alpha \in \Delta} \sup _{b \in \mathcal{B}} \int_{0}^{\infty} l\left(y_{x}(s), \alpha[b](s), b(s)\right) \mathrm{e}^{-\lambda s} \mathrm{~d} s \quad \lambda>0, x \in \bar{\Omega}
$$

and

$$
v(x, t):=\inf _{\alpha \in \Delta} \sup _{b \in \mathcal{B}} \int_{0}^{t} l\left(y_{x}(s), \alpha[b](s), b(s)\right) \mathrm{d} s \quad t \geq 0 x \in \bar{\Omega} .
$$

Recall that by assumptions (2.1) $M$ is an upper bound for $|f(x, a, b)|$. Let $\epsilon>0$ be an arbitrary small (fixed) number. Take $T>0$ and let us chose $\lambda=\frac{\epsilon}{T}$. For any $x \in \bar{\Omega}$, by using the Dynamic Programming Principle, 
we get

$$
\begin{aligned}
\lambda v_{\lambda}(x)= & \inf _{\alpha \in \Delta} \sup _{b \in \mathcal{B}}\left\{\lambda \int_{0}^{T} l\left(y_{x}(s), \alpha[b](s), b(s)\right) \mathrm{e}^{-\lambda s} \mathrm{~d} s+\lambda \mathrm{e}^{-\lambda T} v_{\lambda}\left(y_{x}(T)\right)\right\} \\
= & \inf _{\alpha \in \Delta} \sup _{b \in \mathcal{B}}\left\{\frac{\epsilon}{T} \int_{0}^{T} l\left(y_{x}(s), \alpha[b](s), b(s)\right) \mathrm{d} s\right. \\
& \left.+\frac{\epsilon}{T} \int_{0}^{T}\left(\mathrm{e}^{-\lambda s}-1\right) l\left(y_{x}(s), \alpha[b](s), b(s)\right) \mathrm{d} s+\frac{\epsilon}{T} \mathrm{e}^{-\epsilon} v_{\lambda}\left(y_{x}(T)\right)\right\}
\end{aligned}
$$

and, so, dividing by $\epsilon$ in the equality above, we get

$$
\begin{aligned}
\frac{\lambda}{\epsilon} v_{\lambda}(x)= & \inf _{\alpha \in \Delta} \sup _{b \in \mathcal{B}}\left\{\frac{\epsilon}{T} \int_{0}^{T} l\left(y_{x}(s), \alpha[b](s), b(s)\right) \mathrm{d} s\right. \\
& \left.+\frac{1}{T} \int_{0}^{T}\left(\mathrm{e}^{-\lambda s}-1\right) l\left(y_{x}(s), \alpha[b](s), b(s)\right) \mathrm{d} s+\frac{1}{\epsilon} \lambda \mathrm{e}^{-\epsilon} v_{\lambda}\left(y_{x}(T)\right)\right\} .
\end{aligned}
$$

Now, notice that

$$
\left|\frac{1}{T} \int_{0}^{T}\left(\mathrm{e}^{-\lambda s}-1\right) l\left(y_{x}(s), \alpha[b](s), b(s)\right) \mathrm{d} s\right| \leq \frac{M}{\epsilon}\left(1-\epsilon-\mathrm{e}^{-\epsilon}\right),
$$

and therefore sending $T \rightarrow+\infty$, by the uniform convergence of $\lambda v_{\lambda}$ to $\chi_{0}$ as $\lambda \rightarrow 0^{+}$, we have

$$
-\frac{M}{\epsilon}\left(1-\epsilon-\mathrm{e}^{-\epsilon}\right) \leq \liminf _{T \rightarrow+\infty}\left|\frac{1}{T} v(x, T)-\frac{1-\mathrm{e}^{-\epsilon}}{\epsilon} \chi_{0}\right| \leq \limsup _{T \rightarrow+\infty}\left|\frac{1}{T} v(x, T)-\frac{1-\mathrm{e}^{-\epsilon}}{\epsilon} \chi_{0}\right| \leq \frac{M}{\epsilon}\left(1-\epsilon-\mathrm{e}^{-\epsilon}\right) .
$$

Letting $\epsilon$ go to $0^{+}$we obtain $c$ ) with $\chi_{1}=\chi_{0}$.

$b) \Rightarrow c$ ). We just follow the argument for a construction of a suitable viscosity subsolution (and supersolution) that is given in [2]: actually, what we have to check here is that the Neumann boundary conditions are satisfied for such a function. Choose an arbitrary $\chi$ such that $\chi<\chi_{1}$. Let us consider a smooth function $h:[0,+\infty) \longrightarrow \mathbb{R}$ such that

The function

$$
\left\{\begin{array}{l}
h(0)=0, h^{\prime}(t) \geq \chi \forall t \geq 0 \\
\inf _{x \in \bar{\Omega}} v(x, t)>h(t) \text { for some } t>0 \\
\sup _{x \in \bar{\Omega}} v(x, t)<h(t) \text { for } t \text { large enough. }
\end{array}\right.
$$

$$
w(x):=\sup _{t \geq 0}\{v(x, t)-h(t)\}
$$

is well defined and continuous, moreover, there exists a compact set $K \subset(0,+\infty)$ such that $\forall x \in \bar{\Omega} \exists t_{x} \in K$ with the following property:

$$
w(x)=v\left(x, t_{x}\right)-h\left(t_{x}\right)=\sup _{t \in K}\{v(x, t)-h(t)\} .
$$

It turns out that $w(x)$ is a viscosity subsolution of $\chi+H(x, D w(x))=0$ in $\Omega$ (see [2] for the details). As far as the boundary conditions are concerned, if $x_{0}$ is a local minimum for $w(x)-\phi(x)$ where $\phi$ is a smooth test function, then we get that $\left(x_{0}, t_{x_{0}}\right)$ is a local maximum for

$$
v(x, t)-(h(t)+\phi(x))
$$

where, now, $h(t)+\phi(x)$ is the new test function. Indeed, for any $(x, t)$ close to $\left(x_{0}, t_{x_{0}}\right)$ we have

$$
v\left(x_{0}, t_{x_{0}}\right)-h\left(t_{x_{0}}\right)-\phi\left(x_{0}\right) \geq v\left(x, t_{x}\right)-h\left(t_{x}\right)-\phi(x) \geq v(x, t)-h(t)-\phi(x),
$$


by the definition of $w(x)$ and the properties of $h(x)$. By hypothesis $v(x, t)$ is the viscosity solution of (5.2), and, so, we obtain

$$
\left\langle D\left(h\left(t_{x_{0}}\right)+\phi\left(x_{0}\right)\right), \gamma\left(x_{0}\right)\right\rangle \geq 0
$$

and, hence,

$$
\left\langle D\left(\phi\left(x_{0}\right)\right), \gamma\left(x_{0}\right)\right\rangle \geq 0
$$

Thus, $w(x)$ is a viscosity subsolution of (5.3) and, since $\chi$ is an arbitrary number so that $\chi<\chi_{1}$, we obtain that $\chi_{1} \leq \chi_{S}$. Similarly, one can prove that $\chi_{I} \leq \chi_{1}$ and since $\chi_{S} \leq \chi_{I}$ (see Rem. 5.2), then we have $\chi_{1}=\chi_{I}=\chi_{S}$.

$c) \Rightarrow a$ ). Take a constant $\chi$ such that $v$ is a corresponding subsolution of (5.3); we can assume that $v \leq 0$ by subtracting a suitable constant. Setting $w_{\lambda}:=v+\frac{\chi}{\lambda}$ we have:

$$
\lambda w_{\lambda}+H\left(x, D w_{\lambda}\right)=\lambda v+\chi+H(x, D v) \leq 0 \quad \text { on } \bar{\Omega}
$$

and, moreover, if $x_{0} \in \partial \Omega$ then $\left\langle D w_{\lambda}\left(x_{0}\right), \gamma\left(x_{0}\right)\right\rangle \geq 0$. By using the comparison principle (see Th. 6 in [30]) we have $\lambda w_{\lambda} \leq \lambda v_{\lambda}$. Thus, we get $\liminf _{\lambda \rightarrow 0} \lambda v_{\lambda} \geq \chi$ and, taking the supremum over $\chi$,

$$
\liminf _{\lambda \rightarrow 0^{+}} \lambda v_{\lambda} \geq \chi_{S}
$$

Analogously, we obtain:

and, so, by $c$ ),

$$
\limsup _{\lambda \rightarrow 0^{+}} \lambda v_{\lambda} \leq \chi_{I}
$$

$$
\lim _{\lambda \rightarrow 0^{+}} \lambda v_{\lambda}=\chi_{I}=\chi_{S}
$$

Remark 5.3. If we define the function $w_{\lambda}(x):=v_{\lambda}(x)-v_{\lambda}\left(x_{0}\right)$ where $x_{0} \in \bar{\Omega}$, then we get

$$
\lim _{\lambda_{n} \mapsto 0^{+}} w_{\lambda_{n}}=w_{0} \quad \text { uniformly in } \bar{\Omega}
$$

for some $\lambda_{n} \rightarrow 0^{+}$and $w_{0}=w_{0}(x)$ is a viscosity solution of

$$
\left\{\begin{aligned}
\chi_{0}+H(x, D w) & =0 & & \text { in } \bar{\Omega} \\
\frac{D u}{D \gamma} & =0 & & \text { on } \partial \Omega .
\end{aligned}\right.
$$

Remark 5.4. As far as the state constraints B.C. is concerned, it is possible to prove an analogous equivalence between $a$ ), b) and $c$ ) of the Proposition 5.1 (with $\chi_{0}=\chi_{1}=\chi_{2}$ ), just by using the argument of [2]. In order to prove $a) \Leftrightarrow b$ ) one can the approach based on the representation formula of the viscosity solution and on the Dynamic Programming Principle as in [6] (cf. also [4]).

\section{Appendix: Estimates on the trajectories of the Skorokhod problem}

The appendix is devoted to prove the estimate (3.3) on the trajectories which are solutions of system (3.2). We will follow some ideas of Lions in [29] (see also [30]).

Here, the set $\Omega \subset \mathbb{R}^{n}$ is a domain (not necessarily bounded or connected) such that $\bar{\Omega}$ satisfies the uniform exterior ball condition (see 3.1).

For simplicity of exposition, we prove the case of normal reflection: $\gamma(x)=n(x)$ for any $x \in \partial \Omega$ where $n(x)$ denotes the outer unit normal of $\partial \Omega$. It is possible to prove that analogous results still hold when we consider the Skorokhod problem with oblique reflection, i.e., when instead of the unit outward normal to the boundary, 
$n(x) \forall x \in \partial \Omega$, we have an oblique vector $\gamma(x)$ with the following property: there exists $\nu>0$ such that $n(x) \cdot \gamma(x) \geq \nu \forall x \in \partial \Omega$. The extension to the general case is classical (cf. [29-31]).

Let us start with a easy well-known result.

Lemma 6.1. If $\partial \Omega \in \mathcal{C}^{1}$ and (3.1) holds, then

i) there is a constant $C_{0}>0$ such that $\forall x \in \partial \Omega \forall y \in \bar{\Omega}$ we have

$$
n(x) \cdot(x-y)+C_{0}|x-y|^{2} \geq 0 ;
$$

ii) $\forall x \in \partial \Omega$, if $\exists C \geq 0, \exists \xi \in \mathbb{R}^{n}$ such that $\forall y \in \bar{\Omega}$

$$
\xi \cdot(x-y)+C|x-y|^{2} \geq 0,
$$

then $\xi=\theta n(x)$ for some $\theta \geq 0$.

Proof. The proof is standard: see for instance [29] or [26].

Remark 6.2. Multiplying (6.1) by any constant $\theta \geq 0$, we obtain that $\forall x \in \partial \Omega, \forall y \in \bar{\Omega}$ and $\forall \xi \in N(x):=$ $\{\theta n(x): \theta \geq 0\}$ the following fundamental inequality holds

$$
\xi \cdot(x-y)+C_{0}|\xi||y-x|^{2} \geq 0
$$

where $C_{0}=\frac{1}{2 r}$.

Consider a nonempty set $E \subset \mathbb{R}^{n}$, we denote by $\operatorname{dist}(x, E):=\inf \{|x-y|: y \in E\}$ the distance of $x$ from $E$ on $\mathbb{R}^{n}$.

Let us introduce a regular function on $\bar{\Omega}$, that coincides with the distance from the boundary in a neighborhood of $\partial \Omega$ :

where $\psi$ is a function in $\mathcal{C}^{\infty}([0, \infty), \mathbb{R})$ that satisfies

$$
\tilde{d}(x):=\psi(\operatorname{dist}(x, \partial \Omega))
$$

$$
\left\{\begin{array}{l}
\psi(t)=t \text { if } 0 \leq t \leq \frac{\varepsilon_{0}}{2} \\
0 \leq \psi^{\prime} \leq 1, \text { for } t \geq 0 \\
\psi^{\prime}=0 \text { if } t \geq \varepsilon_{0},
\end{array}\right.
$$

with $\varepsilon_{0}>0$ sufficiently small such that $\operatorname{dist}(x, \partial \Omega)$ is differentiable on the set

$$
\Gamma_{\varepsilon_{0}}:=\left\{x \in \bar{\Omega}: \operatorname{dist}(x, \partial \Omega)<\varepsilon_{0}\right\} .
$$

We get $\psi \equiv \psi_{0} \leq \varepsilon_{0} \forall t \geq \varepsilon_{0}$. We remind that the regularity of the boundary can give sufficient conditions for the required smoothness of $\operatorname{dist}(x, \partial \Omega)$ (see the book [25] or [22]); for example if $\Omega$ is bounded and $\partial \Omega \in \mathcal{C}^{2}$ then $\operatorname{dist}(x, \partial \Omega) \in \mathcal{C}^{2}\left(\Gamma_{\varepsilon_{0}}\right)$ (and the uniform exterior ball condition is also satisfied).

Lemma 6.3. Consider any couple of controls $(a(\cdot), b(\cdot)) \in \mathcal{A} \times \mathcal{B}$ and let $\left(y_{x}(t), L_{t}\right)$ be the solution of (3.2). Then, there exist positive constants $C_{1}$ and $C_{2}$ such that

$$
L_{t} \leq C_{1}+C_{2} t .
$$

Proof. Let $\tilde{d}$ be the function defined by (6.3). We recall that $|\nabla \tilde{d}| \leq 1$ in $\bar{\Omega}$ and $\tilde{d}(x)=d(x, \partial \Omega)$ in a suitable neighborhood of $\partial \Omega$. Just applying the fundamental theorem of calculus (we recall that the controls $a(\cdot)$ and $b(\cdot)$ are chosen)

$$
\tilde{d}\left(y_{x}(t)\right)=\tilde{d}(x)+\int_{0}^{t} f\left(y_{x}(s), a(s), b(s)\right) \cdot \nabla \tilde{d}\left(y_{x}(s)\right) \mathrm{d} s-\int_{0}^{t} \nabla \tilde{d}\left(y_{x}(s)\right) \cdot n(y(s)) 1_{\partial \Omega}\left(y_{x}(s)\right) \mathrm{d} L_{s}
$$


and observing that $\nabla \tilde{d}(x)=\nabla d(x)=-n(x)$ on $\partial \Omega$, we get:

$$
\tilde{d}\left(y_{x}(t)\right)=\tilde{d}(x)+\int_{0}^{t} f\left(y_{x}(s), a(s), b(s)\right) \cdot \nabla \tilde{d}\left(y_{x}(s)\right) \mathrm{d} s+\int_{0}^{t} 1_{\partial \Omega}\left(y_{x}(s)\right) \mathrm{d} L_{s},
$$

and, hence,

Finally we obtain

$$
L_{t}=\tilde{d}\left(y_{x}(t)\right)-\tilde{d}(x)-\int_{0}^{t} f\left(y_{x}(s), a(s), b(s)\right) \cdot \nabla \tilde{d}\left(y_{x}(s)\right) \mathrm{d} s .
$$

where $C_{1}=\varepsilon_{0}$ and $C_{2}=\sup |f(x, a, b) \cdot \nabla \tilde{d}(x)|=M$.

$$
L_{t} \leq C_{1}+C_{2} t
$$

Lemma 6.4. Consider any couple of controls $(a(\cdot), b(\cdot)) \in \mathcal{A} \times \mathcal{B}$. Then the trajectories $y_{x}(\cdot)$ of the Skorokhod problem (3.2) satisfy the estimate (3.3).

Proof. By denoting $L_{t}^{x}=\int_{0}^{t} 1_{\partial \Omega}\left(y_{x}(s)\right) \mathrm{d} L_{s}(x \in \bar{\Omega}$ is the starting point $)$ and recalling that $\left(y_{1}-y_{2}\right) \cdot\left(f\left(y_{1}, a, b\right)-\right.$ $\left.f\left(y_{2}, a, b\right)\right) \leq M\left|y_{1}-y_{2}\right|^{2}$, by the fundamental theorem of calculus we have

$$
\begin{aligned}
\left|y_{x}(t)-y_{z}(t)\right|^{2} \mathrm{e}^{-2 M t-2 C_{0}\left\{L_{t}^{x}+L_{t}^{z}\right\}=} \mid & x-\left.z\right|^{2}+2 \int_{0}^{t}\left[( y _ { x } ( s ) - y _ { z } ( s ) ) \cdot \left(f\left(y_{x}(s), a(s), b(s)\right)\right.\right. \\
& \left.\left.-f\left(y_{z}(s), a(s), b(s)\right)\right) \mathrm{e}^{-2 M s-2 C_{0}\left\{L_{s}^{x}+L_{s}^{z}\right\}}\right] \mathrm{d} s \\
& -2 \int_{0}^{t} \mathrm{e}^{-2 M s-2 C_{0}\left\{L_{s}^{x}+L_{s}^{z}\right\}}\left(y_{x}(s)-y_{z}(s)\right) \cdot\left(n\left(y_{x}(s)\right) \mathrm{d} L_{s}^{x}-n\left(y_{z}(s)\right) \mathrm{d} L_{s}^{z}\right) \\
& -2 M \int_{0}^{t}\left|y_{x}(s)-y_{z}(s)\right|^{2} \mathrm{e}^{-2 M s-2 C_{0}\left\{L_{s}^{x}+L_{s}^{z}\right\}} \mathrm{d} s \\
& -2 C_{0} \int_{0}^{t}\left|y_{x}(s)-y_{z}(s)\right|^{2} \mathrm{e}^{-2 M s-2 C_{0}\left\{L_{s}^{x}+L_{s}^{z}\right\}}\left\{L_{s}^{x}+L_{s}^{z}\right\} \\
\leq & -2 \int_{0}^{t} \mathrm{e}^{-2 M s-2 C_{0}\left\{L_{s}^{x}+L_{s}^{z}\right\}}\left[\left(y_{x}(s)-y_{z}(s)\right) \cdot\left(n\left(y_{x}(s)\right) \mathrm{d} L_{s}^{x}-n\left(y_{z}(s)\right) \mathrm{d} L_{s}^{z}\right)\right. \\
& \left.+C_{0}\left|y_{x}(s)-y_{z}(s)\right|^{2}\left\{L_{s}^{x}+L_{s}^{z}\right\}\right] \leq 0,
\end{aligned}
$$

where the last inequality is given by the property (6.2). Hence, by Lemma 6.3 and the above estimate, we obtain

$$
\begin{aligned}
\left|y_{x}(t)-y_{z}(t)\right| & \leq|x-z| \mathrm{e}^{M t+C_{0}\left\{L_{t}^{x}+L_{t}^{z}\right\}} \\
& \leq|x-z| \mathrm{e}^{M t+2 C_{0}\left(C_{1}+C_{2} t\right)} \\
& \leq K|x-z| \mathrm{e}^{\left(M+2 C_{0} C_{2}\right) t},
\end{aligned}
$$

with $K=\mathrm{e}^{2 C_{0} C_{1}}$.

Acknowledgements. I would like to thank M. Bardi who introduced the subject to me, P. Cardaliaguet for the fruitful discussions and the referee for his precious suggestions.

\section{REFERENCES}

[1] O. Alvarez and M. Bardi, A general convergence result for singular perturbations of fully nonlinear degenerate parabolic PDEs. University of Padova, Preprint (2002).

[2] O. Alvarez and M. Bardi, Singular perturbations of nonlinear degenerate parabolic PDEs: a general convergence result. Arch. Rational Mech. Anal. 170 (2003) 17-61. 
[3] M. Arisawa, Ergodic problem for the Hamilton-Jacobi-Bellman equation I. Ann. Inst. H. Poincaré Anal. Non Linéaire 14 (1997) 415-438.

[4] M. Arisawa, Ergodic problem for the Hamilton-Jacobi-Bellman equation II. Ann. Inst. H. Poincaré Anal. Non Linéaire 15 (1998) 1-24.

[5] M. Arisawa and P.L. Lions, Continuity of admissible trajectories for state constraints control problems. Discrete Cont. Dyn. Systems 2 (1996) 297-305.

[6] M. Arisawa and P.L. Lions, On ergodic stochastic control. Commun. Partial Differ. Equations 23 (1998) $2187-2217$.

[7] J.P. Aubin and A. Cellina, Differential inclusions. Set-valued maps and viability theory. Grundlehren der Mathematischen Wissenschaften [Fundamental Principles of Mathematical Sciences]. Springer-Verlag, Berlin 264 (1984) XIII+342.

[8] M. Bardi and I. Capuzzo-Dolcetta, Optimal control and viscosity solutions of the Hamilton-Jacobi equations. Birkhäuser, Boston (1997).

[9] M. Bardi, S. Koike and P. Soravia, Pursuit-evasion game with state constraints: dynamic programming and discrete-time approximations. Discrete Cont. Dyn. Systems 6 (2000) 361-380.

[10] G. Barles, Solutions de viscosité des équations de Hamilton-Jacobi. (French) [Viscosity solutions of Hamilton-Jacobi equations.] Mathématiques 83 Applications [Mathematics 83 Applications]. Springer-Verlag, Paris 17 (1994) X+194.

[11] P. Bettiol, Weak Solutions in Hamilton-Jacobi and Control Theory. Ph.D. Thesis University of Padova (2002).

[12] P. Bettiol, P. Cardaliaguet and M. Quincampoix, Zero-sum state constrained Differential Games: Victory domains and Existence of value function for Bolza Problem. Preprint SISSA/ISAS Ref. 85/2004/M.

[13] I. Capuzzo-Dolcetta and P.L. Lions, Hamilton-Jacobi equations with state constraints. Trans. Amer. Math. Soc. 318 (1990) 643-687.

[14] P. Cardaliaguet, M. Quincampoix and P. Saint-Pierre, Pursuit differential games with state constraints. SIAM J. Control Optim. 39 (2001) 1615-1632.

[15] P. Cardaliaguet and S. Plaskacz, Invariant solutions of differential games and Hamilton-Jacobi equations for time-measurable hamiltonians. SIAM J. Control Optim. 38 (2000) 1501-1520.

[16] I.P. Cornfeld, S.V. Fomin and Ya.G. Sinal̆, Ergodic theory. Springer-Verlag, New York (1982). X+486.

[17] M.G. Crandall and P.L. Lions, Condition d'unicité pour les solutions généralisées des équations de Hamilton-Jacobi du premier ordre. (French. English summary.) C. R. Acad. Sci. Paris Sér. I Math. 292 (1981) 183-186.

[18] M.G. Crandall and P.L. Lions, Viscosity solutions of Hamilton-Jacobi equations. Trans. Amer. Math. Soc. 277 (1983) $1-42$.

[19] M.G. Crandall, L.C. Evans and P.L. Lions, Some properties of viscosity solutions of Hamilton-Jacobi equations. Trans. Amer. Math. Soc. 282 (1984) 487-502.

[20] L.C. Evans, Partial differential equations. Graduate Studies in Mathematics, 19 AMS, Rhodeisland (1998).

[21] L.C. Evans and H. Ishii, Differential games and nonlinear first order PDE on bounded domains. Manuscripta Math. 49 (1984) 109-139.

[22] H. Federer, Curvature measures. Trans. Amer. Math. Soc. 93 (1959) 418-491.

[23] H. Frankowska, S. Plaskacz and T. Rzeżuchowski, Measurable viability theorems and the Hamilton-Jacobi-Bellman equation. J. Differential Equations 116 (1995) 265-305.

[24] H. Frankowska and F. Rampazzo, Filippov's and Filippov-Ważewski's theorems on closed domains. J. Differential Equations 161 (2000) 449-478.

[25] D. Gilbarg and N.S. Trudinger, Elliptic partial differential equations of second order. Reprint of the 1998 edition. Classics in Mathematics. Springer-Verlag, Berlin (2001). XIV+517.

[26] H. Ishii, Lecture notes on viscosity solutions. Brown University, Providence, RI (1988).

[27] S. Koike, On the state constraint problem for differential games. Indiana Univ. Math. J. 44 (1995) 467-487.

[28] P.L. Lions, Generalized solutions of Hamilton-Jacobi equations, Research Notes in Mathematics. Pitman (Advanced Publishing Program), Boston, Mass.-London 69 (1982) IV+317.

[29] P.L. Lions, Optimal control of diffusion processes and Hamilton-Jacobi-Bellman equations. I. The dynamic programming principle and applications. Comm. Partial Differ. Equ. 8 (1983) 1101-1174.

[30] P.L. Lions, Neumann type boundary conditions for Hamilton-Jacobi equations. Duke Math. J. 52 (1985), 793-820.

[31] P.L. Lions and A.S. Sznitman, Stochastic differential equations with reflecting boundary conditions. Comm. Pure Appl. Math. 37 (1984) 511-537.

[32] P. Loreti and M.E. Tessitore, Approximation and regularity results on constrained viscosity solutions of Hamilton-JacobiBellman equations. J. Math. Systems Estim. Control 4 (1994) 467-483.

[33] B. Simon, Functional integration and quantum physics. Pure Appl. Math. Academic Press, Inc. [Harcourt Brace Jovanovich, Publishers], New York-London 86 (1979) IX+296.

[34] M.H. Soner, Optimal control with state-space constraint. I. SIAM J. Control Optim. 24 (1986) 552-561. 